\title{
Vitreous haemorrhage in massive hemorrhagic polypoidal choroidal vasculopathy: clinical characteristics and surgical outcomes
}

Vitreous hemorrhage in PCV

Raja Narayanan ${ }^{1 *}$, Kopal Mithal ${ }^{1}$, Subhadra Jalali ${ }^{1}$, Jay Kumar Chhablani ${ }^{1}$, Annie Mathai ${ }^{1}$ and Md Hasnat Ali $^{2}$

\begin{abstract}
Background: To report the outcomes of vitreous hemorrhage $(\mathrm{VH})$ associated with hemorrhagic polypoidal choroidal vasculopathy (PCV).

Methods: A retrospective study of 28 eyes of 27 consecutive patients of hemorrhagic PCV with VH, which were managed surgically between January 2003 and December 2011, was performed. All patients underwent pars plana vitrectomy for VH associated with PCV. The main outcome measure was best-corrected visual acuity (BCVA) at baseline, at 1 , 3 and 6 months post operatively and at last follow up.

Results: The visual acuity measured on early treatment diabetic retinopathy study (ETDRS) chart improved in 16 eyes (57.1\%) by two or more lines, remained unchanged in nine eyes (32.1\%) and decreased in three (10.7 \%) after surgery when compared to baseline VA. The mean baseline VA was $2.69 \pm 0.57$ logMAR units $(<20 / 2000)$ which improved to $1.65 \pm 0.93 \log$ MAR units (20/800) at 1 month post operative visit and was sustained at $1.72 \pm 1.12(20 / 800)$ with an improvement of $0.96 \log M A R$ units $(p<0.001,95 \% \mathrm{Cl} 0.54-1.37)$. The average postoperative follow up was for 14.2 months (range 1-84). The complications noted in postoperative follow up were cataract $(n=10)$, macular scaring $(n=9)$, organised dehemoglobinised blood $(n=7)$, retinal tear or detachment $(n=5)$, recurrent VH $(n=3)$ and choroidal detachment $(n=1)$.
\end{abstract}

Conclusion: Majority of patients with loss of vision due to VH secondary to hemorrhagic PCV have sustained improvement in visual acuity following surgery.

Keywords: Polypoidal choroidal vasculopathy, Vitrectomy, Vitreous haemorrhage

\section{Background}

Polypoidal choroidal vasculopathy (PCV) is a persistent and chronic disease with a variable course [1]. Although the prognosis of PCV is commonly reported to be more favorable and the clinical course more stable as compared

\footnotetext{
*Correspondence: narayanan@lvpei.org

1 Srimati Kanuri Santhamma Retina Vitreous Centre, LV Prasad Eye Institute, L V Prasad Marg, Banjara Hills, Hyderabad, Andhra Pradesh 500034 , India

Full list of author information is available at the end of the article This study was presented at the 7th Asia Pacific Vitreoretina Society Meeting, December 14, 2012, Hong Kong.
}

with exudative age-related macular degeneration (AMD) [2-4], the natural history of PCV, especially haemorrhagic $\mathrm{PCV}$, is not clearly known and may not be as benign as previously reported [1].

The primary abnormality in PCV involves the choroidal circulation, and the characteristic lesion is an inner choroidal vascular network of vessels ending in an aneurysmal bulge or outward projection seen clinically as reddish orange polyps [5]. Leakage in the vessel wall at dilatations leads to serous pigment epithelial detachments (PED) and lipid deposition in the exudative form 
of PCV while recurrent serosanguinous detachments of the retinal pigment epithelium and neurosensory retina are the predominant manifestation of hemorrhagic PCV. Massive hemorrhages may develop from rupture of venules and occasionally arteries. Sequelae of this is a sudden onset severe visual loss due to break through vitreous hemorrhage (VH) [1, 6-10].

About half the cases of PCV have a stable course and relatively favorable visual outcome, while the others have persistent leakage, repeated haemorrhages and poor visual outcome [1, 4]. Profound visual loss in hemorrhagic PCV primarily results from recurrent serosanguinous haemorrhagic detachments involving the fovea or recurrent $\mathrm{VH}$.

There is a lack of understanding of hemorrhagic PCV and there are very few reports in the literature on the outcomes of conservative [1, 4], or surgical management of PCV with $\mathrm{VH}[4,6]$. In the current study we report the incidence, clinical characteristics, management and surgical outcome in haemorrhagic PCV presenting with vitreous haemorrhage.

\section{Methods}

This study was a retrospective, interventional consecutive case series. The study was approved by the local ethics committee of L.V. Prasad Eye Institute (LEC-11-160) and all participants gave consent for using patient data for research purpose. Medical records of all cases having a diagnosis ICD coding of PCV and vitreous haemorrhage who underwent pars plana vitrectomy (PPV) were identified and studied. Twenty-eight eyes of 27 consecutive patients treated surgically for PCV associated with $\mathrm{VH}$ were included.

Due to the presence of $\mathrm{VH}$, the media clarity did not allow the visualization of the fundus or angiography in most eyes at presentation. Although the EVEREST trial laid down guidelines for the diagnosis of PCV [11], massive hemorrhagic PCV may not show the same features at presentation in the affected eye. The vitreous haemorrhage was attributed to $\mathrm{PCV}$ on the basis of preoperative B-scan features [6], and confirmed with demonstration of polypoidal lesions and/or branching vascular network in the study or fellow eye on indocyanine angiography (ICGA) in the past or post-operatively, as laid down in the EVEREST trial [11]. Patients were excluded if the same or fellow eye had any other vascular or degenerative retinal or macular condition, or any other ocular pathology which could limit their vision.

After a preoperative written informed consent all patients underwent standard 20 or 23 gauge PPV. Removal of subretinal blood was not attempted in this series.

The data pertaining to age, gender, duration of diabetes and hypertension, duration of symptoms, best-corrected visual acuity (BCVA) at presentation, 1, 3, 6 months postoperative and at the last follow up, intraocular pressure (IOP, Goldman applanation tonometry), surgical procedures performed, surgical complications and duration of follow up were collected and are summarized in Table 1.

The primary outcome measure of this series was the number of patients who improved by two or more lines on early treatment diabetic retinopathy study (ETDRS) chart after surgery. Secondary outcome measures were mean change in logMAR visual acuity, and complications of surgery.

The visual improvement post operatively and the differences between BCVA at 1 month post operative visit and final follow up from the baseline pre operative visual acuity were compared using paired t test. Statistical analysis was done using Statistical software R, version 2.14.1.

\section{Results}

Twenty-eight eyes of 27 patients (21 men, 6 women) with a mean age of $58.89 \pm 13.13$ years (range $36-82$ ) underwent PPV for vitreous haemorrhage associated with PCV between January 2003 and December 2011. Their baseline demographic characteristics are shown in Table 1. Ten (37\%) patients had a history of diabetes mellitus for an average of 13 years (range 3-25 years) and 16 (59\%) patients had a history of hypertension for an average 10.5 years (range $1-36$ ).

\section{Table 1 Demographic and baseline characteristics}

\begin{tabular}{|c|c|c|}
\hline 1. & No of eyes & 28 \\
\hline 2. & Age (mean) & 58.89 years (range $36-82$ ) \\
\hline 3. & Gender & 22 males, 6 females \\
\hline 4. & Bilateral PCV + VH & $4(14.28 \%)$ \\
\hline 5. & DM/duration & $\begin{array}{l}10 \text { patients } / 3 \text { years (range } \\
3-25 \text { years) }\end{array}$ \\
\hline 6. & Hypertension/duration & $\begin{array}{l}15 \text { patients/10.5 years (range } \\
1-36 \text { years) }\end{array}$ \\
\hline 7. & Eye & $13 \mathrm{OD}, 15 \mathrm{OS}$ \\
\hline 8. & $\begin{array}{l}\text { Duration of symptoms in } \\
\text { months }\end{array}$ & 6.2 (range $1-48$ months) \\
\hline 9. & Lens status & $\begin{array}{l}11 \text { phakic, } 6 \text { pseudophakic, } 1 \\
\text { aphakic }\end{array}$ \\
\hline 10. & Previous treatment & $\begin{array}{l}\text { VR surgery }(n=3), \operatorname{IVB}(n=5), \\
\text { PDT }(n=3), \\
\text { Laser }(n=1), \text { IVTA }(n=1) \text {, Oral } \\
\text { steroids }(n=3)\end{array}$ \\
\hline 11. & $\begin{array}{c}\text { Fundus exam at } \\
\text { presentation }\end{array}$ & $\begin{array}{l}\text { No view }(n=21) \text {, hemorrhagic } \\
\text { PED }(n=6) \text {, } \\
\text { subretinal blood }(n=4) \text {, pig- } \\
\text { mentary changes }(n=1)\end{array}$ \\
\hline
\end{tabular}

$P C V$ polypoidal choiroidal vasculopathy, $V H$ vitreous hemorrhage, $D M$ diabetes mellitus, VR vitreoretinal, IVB intravitreal bevacizumab, PDT photodynamic therapy, IVTA intravitreal triamcinolone acetonide, PED pigment epithelial detachment 
The mean duration of symptoms at presentation was $6.2 \pm 3.3$ months with sudden onset profound painless diminution of vision being the most common presenting complaint. The mean preoperative baseline logMAR visual acuity was $2.69 \pm 0.58(<20 / 2000$, range $20 / 400$ to perception of light, PL). At 1 month postoperative follow up, the mean BCVA was $1.69 \pm 0.93$ (20/979, range 20/20 to PL) with a mean improvement of $1.00 \log M A R$ units from the baseline ( $\mathrm{p}<0.001,95 \%$ CI 0.64-1.34). At 3 and 6 months follow up the BCVA was $1.46 \pm 0.93$ (20/576, range 20/20 to $\mathrm{PL})$ and $1.57 \pm 1.26(20 / 743$, range $20 / 20$ to no PL) respectively. The mean BCVA at the last post operative follow up visit was $1.73 \pm 1.14(20 / 1074$, range $20 / 20$ to no PL) with an improvement of $0.96 \log$ MAR units from the baseline ( $\mathrm{p}<0.001,95 \%$ CI 0.54-1.37). The BCVA improved by two or more lines on ETDRS chart in 16 of 28 eyes (57.1\%), stabilized or improved by less than two lines in nine (32.1\%) and worsened in three $(10.7 \%)$ at a mean follow up of 14.28 months. The three eyes which worsened post-operatively had a preoperative vision of hand movement which decreased to PL in one and loss of PL in the other two eyes. The results of visual outcome have been summarized in Table 2. The trend of BCVA change from baseline preoperative to post operative last visit is shown in Fig. 1.

Prior to surgery, five study eyes had been treated with intravitreal bevacizumab, one received intravitreal triamcinolone, three had undergone focal laser treatment, two eyes had undergone sclera buckling in the same eye in the past and three eyes had a history of photo dynamic therapy (PDT). Three patients in whom B-scan showed

\section{Table 2 Surgical management and postoperative outcome}

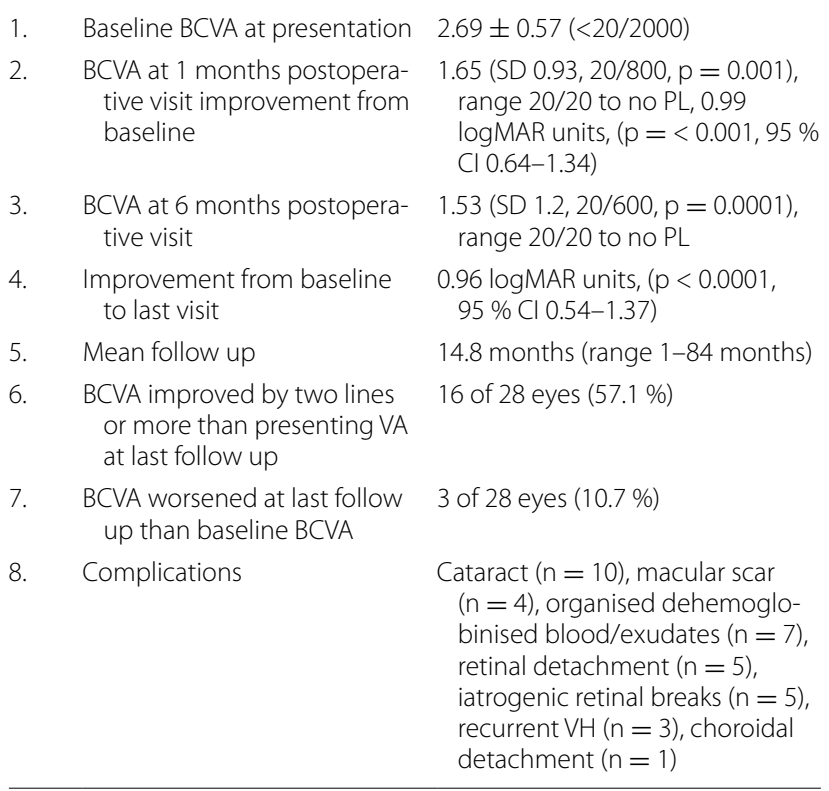

$B C V A$ best-corrected visual acuity, $P L$ perception of light, $V H$ vitreous hemorrhage

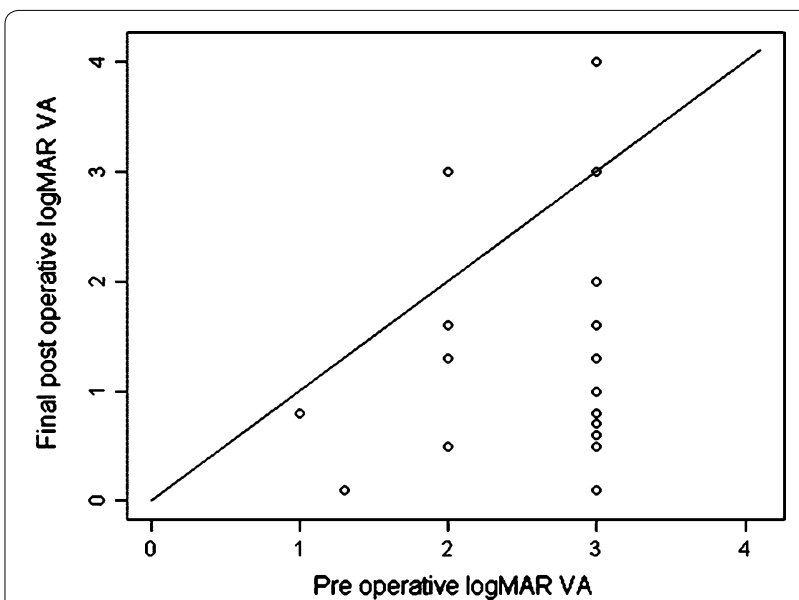

Fig. 1 Scatter plot comparing pre-operative and post-operative best-corrected visual acuity

exudative retinal detachment were treated with oral steroids preoperatively. Nine (33.3\%) patients had bilateral PCV of which four (14.8\%) also had associated VH with $\mathrm{PCV}$ in the fellow eye.

At presentation, fundus evaluation was not possible due to dense vitreous haemorrhage in 21 of the 28 eyes (75\%). B-scan showed vitreous haemorrhage in all 28 eyes, extensive exudative retinal detachment in six and suspected mass lesion in one eye. Diagnosis of underlying cause being PCV was based on demonstration of polyps or branching vascular on previous or post operative ICGAngiography (ICG-A) in seven study eyes, four fellow eyes (Fig. 2) and on fluorescein angiography in two study eyes. The rest of the cases were diagnosed on the basis of presence of multiple sero-sanguinous RPE detachments suggestive of PCV. Two of the cases where polyps had been demonstrated on ICG-A before $\mathrm{VH}$ had regression of the previously noted polyps, but developed polyps in new locations subsequently (Fig. 3).

The site of subretinal haemorrhage was found to be predominantly macular in $12(42.8 \%)$ eyes, peripapillary in 4 (14.2\%) eyes, midperipheral in 10 (35.7\%) eyes and peripheral in 1 (3.5\%) eye. Intraoperatively, macular scar was noted in 9 (32.1\%) eyes and organized dehemoglobinized blood at the macula was noted in seven $(25 \%)$ eyes.

The most common surgical complication was iatrogenic retinal break which was seen in 5 (17.8\%) eyes, which occurred typically during induction of posterior vitreous detachment (PVD). Postoperatively, 10 (35.7 \%) eyes developed visually significant cataract, 3 (10.7\%) eyes developed recurrent vitreous haemorrhage, 5 (17.8\%) had retinal detachment and 1 (3.5\%) patient had choroidal detachment. The mean IOP at baseline was $13.64 \pm 2.52$ and $13.57 \pm 4.12 \mathrm{~mm} \mathrm{Hg}$ at the last follow up. 


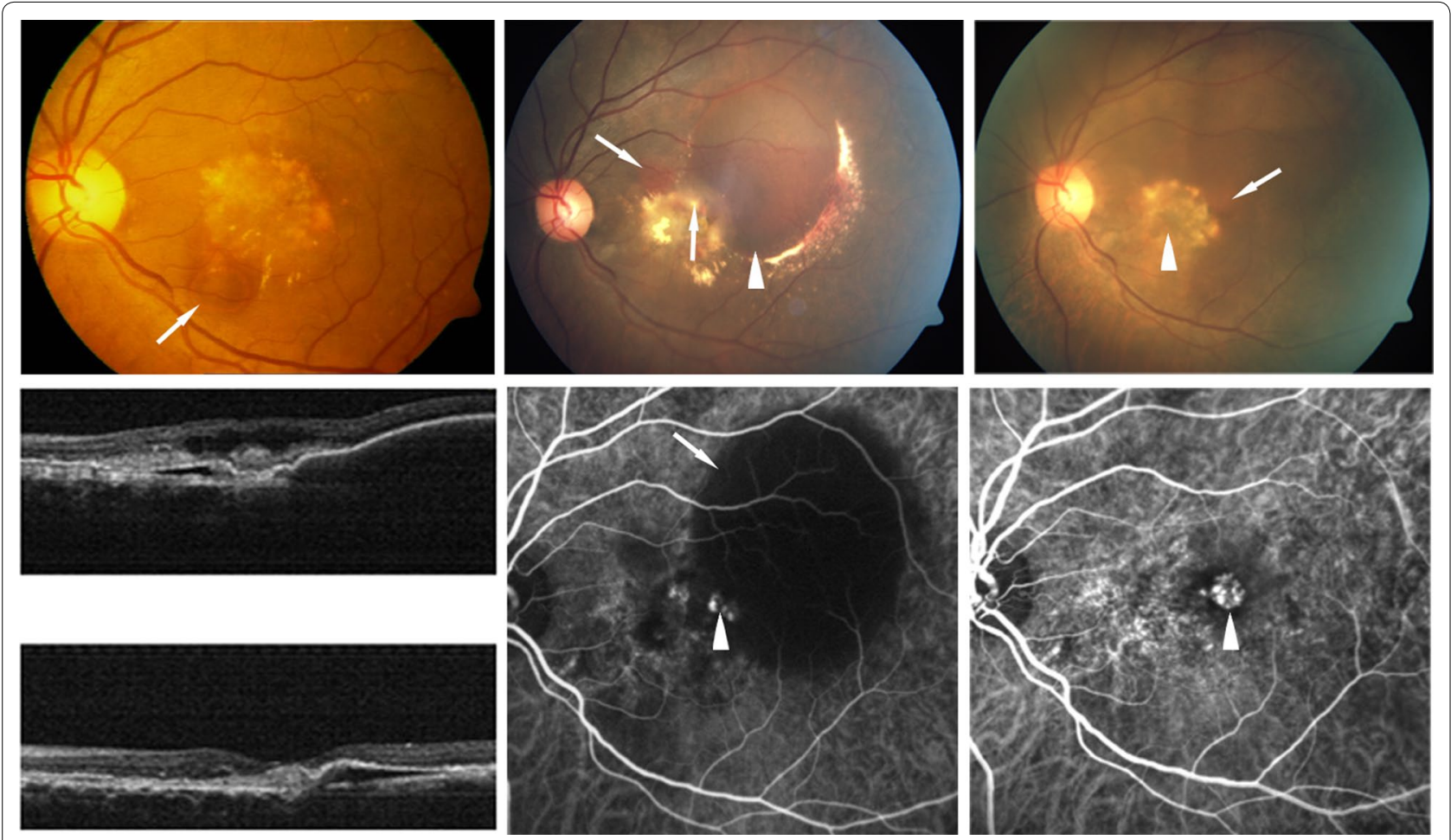

Fig. 2 Top row shows polypoidal lesions (white arrows) demonstrated on indocyanine green angiography (ICG-A) in three study eyes done postoperatively after clearing of vitreous hemorrhage. Bottom row shows ICG-A of fellow eyes in three cases where the ocular media in the study eyes did not clear postoperatively to allow an angiography and the basis of diagnosis was demonstration of polypoidal lesions (white arrows) and/or choroidal branching vascular network (white arrow heads) in the fellow eye

\section{Discussion}

Several reports and case series describing the diverse clinical spectrum, natural course, and role of various modalities of treatments for exudative PCV have been published in the last three decades $[1,2,6-9,11,12]$, but there are few reports in the literature on the management of vitreous haemorrhage associated with $\operatorname{PCV}[6,8,9$, 13]. Vitreous haemorrhage associated with PCV differs from other manifestations of PCV as these eyes are not amenable to ICG angiography. Unless the affected eye has been previously diagnosed as PCV or the fellow eye has features of PCV, it is difficult to diagnose them. B-scan features that can differentiate vitreous haemorrhage secondary to PCV from other causes has been reported and are very useful in this situation. B-scan can demonstrate defining features like blood lining an elevated membrane, hemorrhagic pigment epithelial detachment and absence of any acoustic shadowing. The natural course or role of any treatment modality is unknown for vitreous haemorrhage in PCV as no large series of cases has been studied. In our study, PCV was bilateral in 9 (33.3 \%) of which four eyes (14.8\%) also had PCV associated vitreous haemorrhage in the fellow eye. Previously, bilaterality has been reported ranging from 9 to $47 \%$ for exudative
PCV $[1,8,9,12,14]$. PCV had been originally reported in hypertensive middle aged African women [8, 14-16]. Associated systemic hypertension has been reported in $18-88 \%$ in various case series and clinic pathological studies of PCV $[8,9,14]$. In our series, $59 \%$ of the patients had systemic hypertension.

Vitreous haemorrhage associated with PCV has also been reported to occur following PDT [7, 17]. In our series only one patient had history of recent PDT and developed sudden onset loss of vision and VH 1 week after the treatment.

The earliest descriptions of vitreous haemorrhage in PCV was in $1985[9,10]$. In previous reports by Kleiner et al. and Perkovich et al. $[8,9]$, visual recovery after vitrectomy was found to be equivocal. Two of the three eyes gained useful vision in one series [8], whereas the other studies reported worsening or no change in the visual acuity $[4,9]$. These studies had small series ranging from one to three eyes that underwent vitrectomy. Recent reported outcomes of vitrectomy in PCV did not find a higher incidence of retinal breaks $[13,18]$.

In our study, significant visual improvement occurred in 16 of 29 eyes $(57.1 \%)$. Three patients recovered a visual acuity of better than $20 / 40$, one eye had a visual 

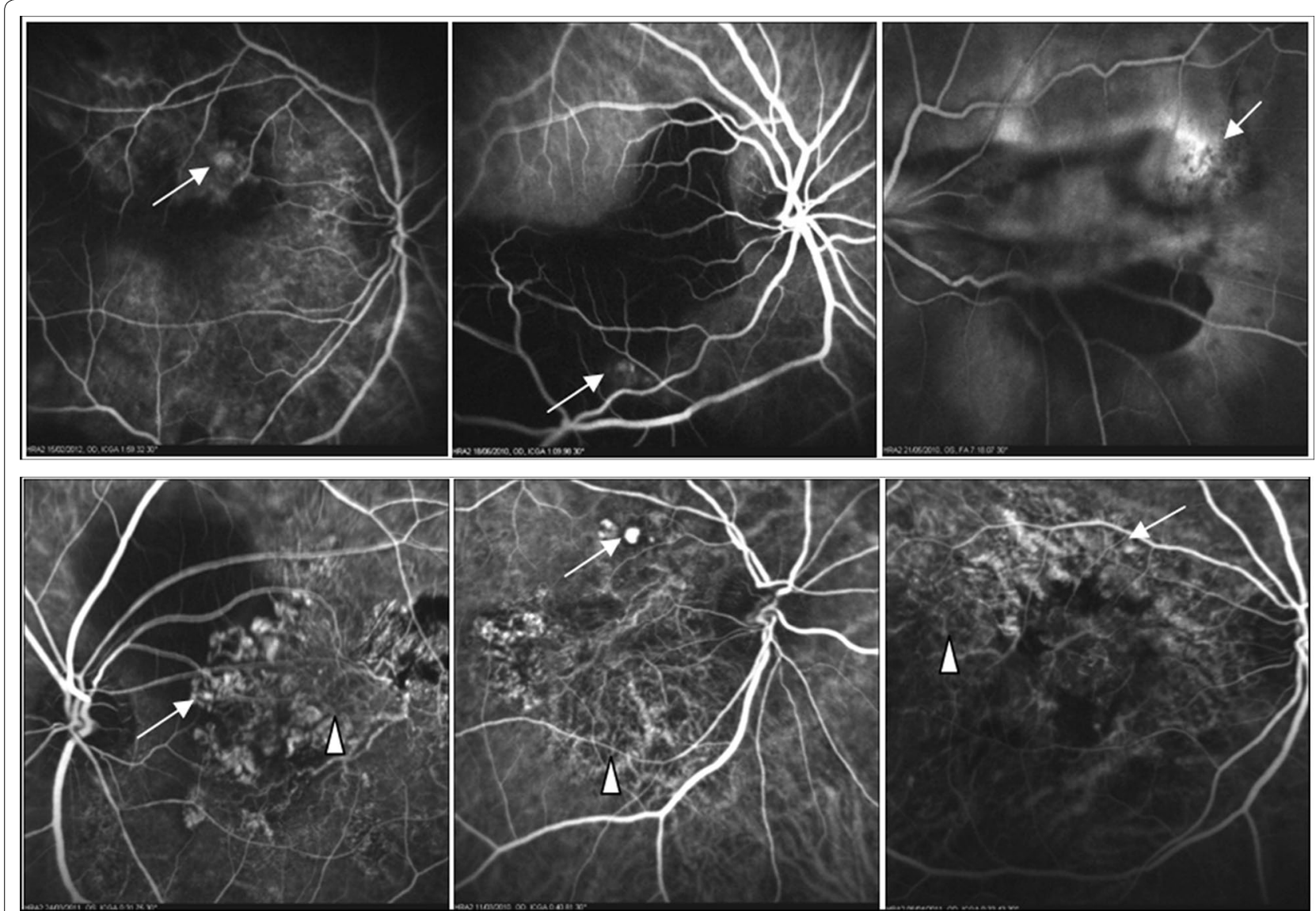

Fig. 3 Both eyes of a 60 year old male with bilateral polypoidal choroidal vasculopathy (PCV) associated with vitreous hemorrhage (VH). Left top picture shows the fundus picture of the left eye at baseline before VH showing serous pigment epithelial detachment (PED, white arrow) and subretinal old blood. Top centre picture shows hemorrhagic pigment epithelial detachment (white arrowhead) and elevated orange yellow lesion before VH (white arrow). Top right picture shows fundus of the left eye post vitrectomy showing macular scar (white arrowhead), disappearance of previously seen polyp and development of a new orange elevated lesion (white arrow). Middle right indocyanine green angiography (ICG-A) of the left eye shows polypoidal lesions (white arrowheads) before VH. Middle centre shows ICG-A of the left eye after vitrectomy for VH shows disappearance of polyps and scar alone (white arrowhead). Middle right shows preoperative and postoperative OCT showing improvement of PED and resolution of intraretinal fluid. Bottom right fundus picture shows PED (white arrowhead). Bottom centre shows ICG angiography done before development of VH shows a polypoidal lesion (white arrow) and a PED (white arrowhead) and bottom left picture shows serosanguinous retinal detachment (arrow)

acuity of $20 / 20$ at 84 months, another had $20 / 25$ at 17 months and the third eye had 20/40 at 6 months follow up. The improvement in visual acuity could have been limited by extensive blood under the macula. We did not attempt to remove the subretinal blood in our study. All cases in our study would have required subretinal tissue plasminogen activator with incubation of approximately half an hour. In our experience, the blood decolorizes rapidly in these cases and does not liquefy with conventional doses of tPA. In such cases, the dose of tPA is much more than the recommended upper limit of $50 \mu \mathrm{g}$. Further, washing of blood in such massive hemorrhage under the macula tends to cause photoreceptor damage. The peripheral visual field would also have improved after the clearance of vitreous haemorrhage but would be difficult to document in most cases due to poor central fixation.

The commonest intra operative complication was iatrogenic retinal breaks, which occurred during induction of PVD which was also been reported by us in our previous study [6]. Hence it is possibly better to wait for spontaneous PVD to occur before attempting surgery. The cases which had no visual recovery or experienced worsening of vision had macular scar, recurrent $\mathrm{VH}$, post operative retinal detachment and cataract.

In a few cases in our study, polypoidal lesions which were seen on ICG-A before vitreous haemorrhage were not seen postoperatively but new lesions polypoidal lesions developed in a new location on subsequent follow up (Fig. 3). This disappearance of classic polypoidal 
lesions and replacement by RPE atrophy and scarring has been described in previously reported cases $[1,9]$. The development of the haemorrhagic PEDs from the preexisting polypoidal lesions, followed by breakthrough $\mathrm{VH}$ and then subsequent scarring may be the natural course of the condition.

\section{Conclusion}

Our study shows that vitrectomy can produce significant visual improvement in patients with vitreous haemorrhage due to PCV. The incidence of retinal breaks during vitrectomy is high in PCV, and caution should be exercised while inducing PVD in such cases.

\begin{abstract}
Abbreviations
AMD: age-related macular degeneration; BCVA: best-corrected visual acuity; ETDRS: early treatment diabetic retinopathy study; ICGA: indocyanine angiography; IOP: intraocular pressure; PPV: pars plana vitrectomy; PL: perception of light; PDT: photo dynamic therapy; PED: pigment epithelial detachments; PCV: polypoidal choroidal vasculopathy; PVD: posterior vitreous detachment; VH: vitreous hemorrhage.
\end{abstract}

\section{Authors' contributions}

Design and conduct of the study (RN), collection, management, analysis, and interpretation of the data ( $\mathrm{RN}, \mathrm{KM}, \mathrm{HA})$, and preparation, review, or approval of the manuscript (RN, SJ, JC, ANM, KM). All authors read and approved the final manuscript.

\section{Author details}

1 Srimati Kanuri Santhamma Retina Vitreous Centre, L V Prasad Eye Institute L V Prasad Marg, Banjara Hills, Hyderabad, Andhra Pradesh 500 034, India. ${ }^{2}$ Department of Biostatistics, Kallam Anji Reddy Campus, LV Prasad Eye Institute, Hyderabad, India.

\section{Acknowledgements}

None.

\section{Competing interests}

The authors declare that they have no competing interests.

\section{Funding}

This research received no specific grant from any funding agency in the public, commercial or not-for-profit sectors.

Received: 14 August 2015 Accepted: 2 November 2015

Published online: 20 December 2015

\section{References}

1. Uyama M, Mitsumasa W, Nagai Y, Matsubara T, et al. Polypoidal choroidal vasculopathy: natural history. Am J Ophthalmol. 2002;133:639-48.

2. Yannuzzi LA, Ciardella A, Spaide RF, et al. The expanding clinical spectrum of idiopathic polypoidal choroidal vasculopathy. Arch Ophthalmol. 1997; 115:478-85.

3. Moorthy RS, Lyon AT, Rabb MF, et al. Idiopathic choroidal vasculopathy of the macula. Ophthalmology. 1998;105:1380-5.

4. Lip P, Hope-Ross MW, Gibson JM. Idiopathic polypoidal choroidal vasculopathy: a disease with diverse clinical spectrum and systemic associations. Eye. 2000;14:695-700.

5. Ciarcella AP, Donsoff IM, Huang SJ, Costa DL, Yannuzzi LA. Polypoidal choroidal vasculopathy: major review. Surv Ophthalmol. 2004;49:25-37.

6. Jalali S, Parra SL, Majji AB, Hussain N, Shah V. Ultrasonographic characteristics and treatment outcomes of surgery for vitreous hemorrhage in idiopathic polypoidal choroidal vasculopathy. Am J Ophthalmol. 2006;142:608-19.

7. Rishi P, Kadekar A, Rishi E. Breakthrough vitreous hemorrhage after ICGA guided PDT for PCV. Indian J Ophthalmol. 2009;57:160-1.

8. Perkovich BT, Zakov ZN, Berlin LA, et al. An update on multiple serosanguinous retinal pigment epithelial detachments in black women. Retina. 1990;10:18-26.

9. Kleiner RC, Brucker AJ, Johnston RL. The posterior uveal bleeding syndrome. Retina. 1990;10:9-17.

10. Stern RM, Zakov ZN, Zegerra H, et al. Multiple recurrent serosanguineous retinal pigment epithelial detachments in black women. Am J Ophthalmol. 1985;100:560-9.

11. Shiraga F, Matsuo T, Yokoe S, et al. Surgical treatment of submacular hemorrhage associated with idiopathic polypoidal choroidal vasculopathy. Am J Ophthalmol. 1999;128:147-54.

12. Yamaoka S, Okada AA, Sugahara M, Hida T. Clinical features of polypoidal choroidal vasculopathy and visual outcomes in the absence of classic choroidal neovascularisation. Ophthalmologica. 2010;224:147-52.

13. Jung JH, Lee JK, Lee JE, Oum BS. Results of vitrectomy for breakthrough vitreous hemorrhage associated with age-related macular degeneration and polypoidal choroidal vasculopathy. Retina. 2010;30(6):865-73.

14. Ahuja RM, Stanga PE, Johannes RV, Rech AC, Bird AC. Polypoidal vasculopathy in exudative and hemorrhagic pigment epithelial detachments. Br J Ophthalmol. 2000;84:479-84.

15. Ross RD, Glitter KA, Cohen G, Shomaker KS. Idiopathic polypoidal choroidal vasculopathy associated with retinal macroaneurysm and hypertensive retinopathy. Retina. 1996;16:105-11.

16. Yannuzzi LA, Sorenson J, Spaide RF, Lipson B. Idiopathic polypoidal choroidal vasculopathy (IPCV). Retina. 1990;10:1-8.

17. Ojima Y, Tsujikawa A, Otani A, Hirami Y, Aikawa H, Yoshimura N. Recurrent bleeding after photodyanamic therapy in polypoidal choroidal vasculopathy. Am J Ophthal. 2006;141:958-60.

18. Lin HC, Yang CH, Yang CM. Visual outcomes of vitrectomy for polypoidal choroidal vasculopathy-related breakthrough vitreous haemorrhage. Eye (Lond). 2014;28(7):797-806.

Submit your next manuscript to BioMed Central and we will help you at every step:

- We accept pre-submission inquiries

- Our selector tool helps you to find the most relevant journal

- We provide round the clock customer support

- Convenient online submission

- Thorough peer review

- Inclusion in PubMed and all major indexing services

- Maximum visibility for your research

Submit your manuscript at www.biomedcentral.com/submit
( Biomed Central 